digestive impairment should, I think, be classed as "complicated," to avoid the necessity of too many criteria in classification. These digestive disturbances are rather in: definite; they vary within a short space of time, are markedly influenced by treatment, and cannot easily be divided into degrees of severity. For these reasons I think slight digestive disturbances should be ignored in classification, severe or persistent disturbance of this kind reckoned as a complication.

\section{Remarks on Methods.}

Precautions as to temperature taking. - In order to avoid the disturbing effects of the journey to the sanatorium or health resort or of occupation, it is advisable to keep patients in bed in every case for the first week, as already done in many sanatoria, and to disregard the first three days. The case would be classified according to the maximum temperature in the last four days of the first week. During these days, at all events, the temperature should be recorded not less than four times a day.

The method of taking temperatures should always be stated. Personally, I am of opinion that for such purposes rectal thermometry is necessary, and it would be as well to adopt a uniform time of five minutes, irrespective of the kind of thermometer used, as this would ensure a greater degree of accuracy with relatively insensitive thermometers. If it is not possible, for any reason, to take the temperature per rectum, and mouth temperatures are taken, we must give time for the mouth cavity to be warmed up, and prevent loss of heat by wrapping up the face, the thermometer being retained for 20 minutes without opening the mouth. No temperature should be taken within one hour of food-taking in the case of mouth temperatures, or within half an hour of defæcation in the case of rectal temperatures.

The difference between rectal and mouth temperatures is a variable one, being less at higher temperatures than at lower, greater in winter than in summer, more in one patient than in another. Only rectal temperatures should be taken on test days to determine the classification of the case. Temperatures taken in the stream of urine are unreliable without safeguards which cannot usually be enforced. Those physicians who dislike rectal thermometry, and find mouth temperatures a sufficient guide to treatment, need only take rectal temperatures on test days.

Putse-rate.-This should only be recorded after at least half an hour without conghing fit or ablutions or exertion of any kind.

Weights.-In estimating weights, the weight of the clothes should always be deducted. The standard adopted should be either the highest known weight in health or, if this is not known, the average for height, age, and sex. Loss of weight being much less important than increase in fever or pulse-rate, only considerable losses have been taken into account in this classification.

\section{Results of Treatment.}

In recording improvement or the reverse, I would suggest that patients staying six weeks or less be classed apart. So short a period of treatment can only as a rule be regarded as educational, not curative. Patients longer under treatment might be grouped according as they have been treated for three months or less; over three and under six months; over six and up to 12 months; or over 12 months.

Constitutional and local results should be stated separately. Dr. C. T. Williams pointed out the advisability of this years ago; and the adoption of the classification suggested in this paper would give a convenient basis for such statements. Whenever the patient has not been long enough mnder treatment to ensure arrest of the disease, removal from Class B to Class A might be regarded as evidence of moderate improvement, from Class $\mathrm{C}$ to Class $\mathrm{A}$ as marked improvement, lesser degrees of improvement being recorded as slight. As regards local improvement, disappearance of adventitious sounds, even on cough, from an area the extent of one lobe (provided this be not caused by blocking of bronchial tubes) might indicate marked improvement, from half a lobe moderate improvement, smaller degrees slight improvement. Patients remaining long enough for apparent arrest should pass a test modelled on that of the American Medical Association-riz, absence of constitutional symptoms of disease beyond such dyspnœea as is inseparable from loss of lung tissue. permanent absence of râles and crepitations even on cough, free entry of air everywhere, and absence of expectoration ever containing tubercle bacilli. Continuance of these conditions for a twelvemonth to indicate apparent oure.

Ultimate results. - There are many fallacies in classifying the ultimate results of treatment of lung disease. Seeing that the disease is caused in many cases by unfavourable conditions or habits of life, it is illogical to apply the same test to one who returns to an unhealthy employment and to another who goes back to favourable conditions. Indeed, when the patient has not reached apparent cure, the results: might more logically be classified according to conditions of life than according to treatment, since the former continue much longer and often have more influence on the final result. A full discussion of the methods of recording results of treatment in lung disease would, however, take too long, and must be left for some future communication.

Bibliography-1. Turban : The Diagnosis of Tuberculosis of the Lung, Trans., E. C. Morland, 1905. 2. Tubereulosis, Berlin, 1907, Band vi., p. 559. 3. Ibid.. Band vi., p. 140. 4. Die Hanclhabung des Heilverfahrens bei Versicherten im Jahre 1896, Hamburg, 1897. 5. Lawrason Brown: The Classification of Pulmonary Tuberculosis, Journal of the American Medical Association, vol. lii., No. 5, Jan. 30th, 1909.

Farnhan.

\section{Cliniral a}

\section{MEDICAL, SURGICAL, OBSTETRICAL, AND THERAPEUTICAL.}

\section{NOTE ON A CASE OF RECURRING ATTACKS OF INTUSSUSCEPTTON.}

By Thomas H. Kellock, M.C. Cantab., F.R.C.S. Eng., SURGEON TO THE MIDDLESEX HOSPITAL AND TO THE HOSPITAI FOR SICK CHILDREN, GREAT ORMOND-STREET, W.C., ETC.

OccURRING, as it generally does, in well-nourished and thriving infants, intussusception would appear to be an affection of the healthy rather than of the unhealthy or delicate, and it is therefore the more to be wondered at that recurrence after reduction is so rare. 'That it does not return immediately is easily explained by the cedema of the in. tussusceptum, which renders reduction difficult and immediate recurrence unlikely, but when the cedema has completely subsided, as it probably does very shortly after reduction, it is rather difficult to explain why the same conditions as brought about the original trouble should not become reestablished and cause a recurrence. Almost everyone who has written on the subject mentions this rarity of recurrence, and it is probably an uncommon experience to be called upon to operate more than once on the same patient for this affection. The following case is of some interest in this connexion, especially as it is now nearly seven years since the first operation. 'The patient has recently been under my care again for an acute glandular abscess in the neck, and it seemed that his previous experience would be worth recording.

The child's history is as follows. Born at full term in January, 1904, he was breast-fed and quite healthy until the age of 10 months, when, just as he was commencing to have other food, he was one day seized with an attack of abdominal pain, vomited frequently, and passed blood and slime from the rectum. After four days of this condition intussusception was diagnosed and he was taken to an hospital, where he was successfully treated by inflation with air. Five months later he had a precisely similar attack after being perfectly well during the interval. An anæsthetic was given and the bowel injected with warm water. This was apparentily quite successful, for the child was quite well during the next six months, and then (October, 1905) another attack occurred, and he was admitted under my care at the Hospital for Sick Children, Great Ormond-street.

On admission the child showed the usual signs of acute intussusception -romiting, the passage of blood and mucus from the rectum, and the presence of an elongated swelling in the right iliac region. The abdomen was opened through the right rectus muscle and an intussusception was found occupying the last 8 inches of the ileum, but not passing through the ileo-cæcal valve. This was easily reduced, although the entering bowel was a good deal thickened by 
congestion. No adhesions were found, but the lymphatic glands in the mesentery corresponding to the intussuscepted portion of the bowel were much enlarged. Recovery was uneventful, and the child was sent home.

Four months later (March, 1906), whilst at home, the child had what appeared, from the history given later by the mother, to be a typical attack of intussusception, during which blood and mucus were passed by the rectum. From this he recovered spontaneously, and when seen at the hospital a few days later nothing abnormal conld be detected. One month later (April, 1906) what wonld seem to be the fifth attack occurred, when in the early morning he was seized with abdominal pain, was sick four or five times during the day, bringing up a little blood on one occasion, and in the afternoon passed blood and mucus by the rectum. In the evening of the same day he was again admitted to the hospital under my care. On admission he is stated to have been a fat and healthy looking child, but somewhat drowsy; temperature $96.4 \circ \mathrm{F}$; ; abdomen flaccid, not tender; a definite tumour could be felt in the right iliac region passing into the right loin. Shortly after admission the abdomen was again opened, on this occasion by splitting the muscles in the right iliac region. An ileo-colic intussusception was then found about 5 inches in length, the lower end of the ileum being prolapsed through the ileo-cæcal valve. Reduction was more difficult than on the previous occasion, as the intussusception was very cdematous and the mesenteric glands much enlarged, soine to $1_{\frac{1}{2}}$ inches in length. After reduction had been effected three silk sutures were inserted into the mesentery of the lower part of the ileum, plicating and shortening it. Recovery was again uninterrupted and the child was sent home three weeks later.

Six years have now elapsed and the child has during that time had no return of abdominal trouble. He has grown into a fairly healthy-looking boy, still showing the marks of the two operations, but without any hernial protrusion at their sites. His experience of intussusception and its treatment is probably unique, for he was treated by inflation with air, injection with water, abdominal section twice, and on one occasion recovery seems to have been spontaneous. What share the plicating of the mesentery at the second operation took in preventing recurrence must, of course, be doubtful, for the tendency to intussusception diminishes as age advances, and so it may be that no further attacks wonld have occurred had this not been done. Still, its performance coincided with the termination of a rather unusual series of attacks of this affection.

Queen Anne-street, $W$.

\section{A NOTE ON INTRACTABLE OTORRHEA DUE TO LATENT EMPYEMA OF THE ANTRUM OF HIGHMORE.}

By Nert C. Scott, M.B., Ch.B. Glasg., HOUSE SURGEOX, LONDON THROAT HOSPITAL.

Mr. Herbert Tilley, in his book on "Diseases of the Nose and Throat," says: "I have seen two cases of intractable otorrhcea immediately clear up when the corresponding maxillary antrum was drained of its septic contents." I have recently seen two such cases, and I think it well to draw attention to the course of the otorrhoea, which is peculiar and, I think, diagnostic.

CASE 1. - A man, aged 23, had acute right-sided suppurative otitis media following a cold in the head. This at first ran the usual course. The discharge became scanty and purulent, but just when one expected it to cease and the perforation to heal, there was a relapse to the profuse serous discharge. The ear sometimes remained dry for a few days, but never more than a fortnight. This continued for six months in spite of treatment. The patient also complained of an intermittent sense of discomfort in the other ear. The perforation was in the anterior segment of the membrana tympani and central. 'The hearing varied but was fairly good. Pus was then discovered in the right antrum of Highmore which was drained by removing the antro-nasal wall. The cavity was irrigated daily with boric lotion. In four weeks the discharge from the ear ceased, and now, four months later, it has not recurred. The perforation in the membrana tympani is now much smaller and the hearing has improved. The empyema of the antrum probably dated from acute rhinitis three years before, but had caused so little inconvenience that its presence was not suspected. There was slight hypertrophic rhinitis and the patient had had to hawk up a few pellets of tough muco-pus from the nasopharynx every morning.

CASE 2.-A man, aged 32, had discharge from the right ear on and off for six years. The otorrhoa had never ceased for more than three weeks at a time. He also complained of intermittent discomfort in the other ear and of hawking up phlegm from the throat. On examination there were hypertrophic rhinitis and a fringe of polypi on the right middle turbinal. There was some muco-pus in the nasopharynx and some granular pharyngitis. The ear and nose were treated for a considerable time without improvement. Pus was then found in the right maxillary antrum, and a radical operation was performed through the canine fossa and the nose. The otorrhœa ceased immediately, and now, nine weeks later, has not recurred.

To sum up, I think an otitis media showing the following characteristics should lead to a careful examination of the corresponding antrum of Highmore: (1) Intermittent otorrhœa; (2) central perforation of the membrana tympani; (3) hearing varying from time to time, but is better than one would expect after prolonged suppuration; and (4) intermittent discomfort in the other ear.

One must remember that an empyema of the antrum of Highmore may be present for years and cause practically no inconvenience. As Mr. Lack says in his book on "Diseases of the Nose," "Cases of antral suppuration are frequently met with in which the patient complains only of hawking up phlegm from the throat, and will not admit that he has any nasal discharge, even on cross-examination."

Great Portland-street, $\mathrm{W}$.

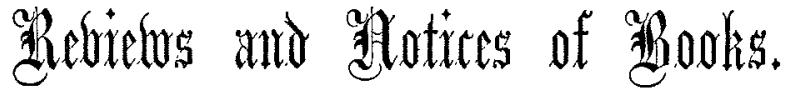

Principles of Human Physiology. By EnNest H. STARLING, M.D. Lond., F.R.C.P. Lond., F.R.S., Jodrell Professor of Physiology in University College, London. With 564 figures in the text. London: J. and A. Churchill. 1912. Pp. 1423. Price 21s. net.

THE present time seems to the author fitting for the production of a text-book of physiology which, while not neglecting the data of physiology, should lay stress upon the mutual stimulation and coopperation among the different sciences, especially in the regions of physics and chemistry; and should attempt to weave these data into a fabric representing the principles that are guiding physiologists and physicians of the present day in their efforts to extend the bounds of the known and to increase their powers of control over the functions of living organisms. The mere preliminary reading of some primer on physiology is not in our opinion sufficient to enable the student to follow much that Professor Starling sets forth with such lucidity. Readers of this volume should come to its study with a mind well stored with the leading facts in physics and chemistry. Purposely the author has omitted a full account of the multifarious methods employed in the experimental investigation of the different organs of the human body. He shows that the only foundation for rational therapeutics is the proper understanding of the working of the human body. Until we know more about the physiology of nutrition "quacks will thrive and food faddists abound." "Ignorance of physiology tends to make a medical man as credulous as his patients, and almost as easily beguiled by the specious puffings of the advertising druggist."

The work is divided into four books of unequal length: I., General Physiology; II., Mechanisms of Movement and Sensation ; III., Mechanisms of Nutrition; and IV., Reproduction. In Book I. the general concepts of the science are set forth, and the student is advised to omit it on a first perusal of the text-book. In it the structural, material, and 